Arch Pharm Res Vol 33, No 6, 967-968, 2010

\title{
Erratum
}

Correction to "The Analgesic and Anti-inflammatory Effects of 7-Oxosandaracopimaric Acid Isolated from the Roots of Aralia cordata"

The article

Arch Pharm Res Vol 33, No 4, 509-514, 2010

\section{The Analgesic and Anti-inflammatory Effects of 7-Oxosandara- copimaric Acid Isolated from the Roots of Aralia cordata}

\author{
Tae Doo Kim, Ji Yun Lee, Bong Jae Cho, Tae Wook Park, and Chang Jong Kim \\ Division of Pathophysiology and Pharmacology, College of Pharmacy, Chung-Ang University, Seoul 156-756, Korea
}

On page 510, right column, the first paragraph in the "Isolation of 7-oxosandaracopimaric acid (OSA) from ACR" mentions:

Original wording:

Compounds 2 was identified as 7-oxosandaracopimaric acid (Fig. 1) by comparing its spectral data with previous reports (Esquivel et al., 1987; Chang et al., 2000, 2008).

This should be replaced by:

Compounds 2 was identified as 7-oxosandaracopimaric acid (Fig. 1) by comparing its spectral data with previous reports (Esquivel et al., 1987; Zinkel and Petersen, 1987; Chang et al., 2000, 2008).

In connection to this, the following article should be added to the References section:

Zinkel, D. F. and Petersen, R. C., Streochemistry of the Tetrahydroisopimaric Acids: X-ray Structure of methyl 8- $\alpha$-Isopimaran-18-olate. J. Chem. Soc. Perkin Trans. I, 1975-1977 (1987).

On page 511, left column, the first paragraph in the "7-oxosandaracopimaric acid (OSA)", the ${ }^{1} \mathrm{H}-\mathrm{NMR}$ and ${ }^{13} \mathrm{C}-\mathrm{NMR}$ data should be corrected as follows:

Original wording:

7-oxo-sandaracopimaric acid (OSA)

Colorless needles: $m p$ 247-249 ${ }^{\circ} \mathrm{C}$, EI-MS $m / z$ : $316.4[\mathrm{M}+],{ }^{1} \mathrm{H}-\mathrm{NMR}\left(500 \mathrm{MHz}\right.$, pyridine- $\left.d_{5}\right): \delta 6.81(1 \mathrm{H}, \mathrm{s}, \mathrm{H}-14), 5.67(1 \mathrm{H}$, dd, $J=10.2,16.2 \mathrm{~Hz}, \mathrm{H}-15), 5.02(1 \mathrm{H}, \mathrm{dd}, J=1.4,10.2 \mathrm{~Hz}, \mathrm{H}-16 \mathrm{~b}), 4.80(1 \mathrm{H}, \mathrm{dd}, J=1.4,16.2 \mathrm{~Hz}, \mathrm{H}-16 \mathrm{a}), 3.72(1 \mathrm{H}, \mathrm{dd}, J$ $=13.6,19.2 \mathrm{~Hz}, \mathrm{H}-6 \mathrm{~b}), 3.13(1 \mathrm{H}, \mathrm{dd}, J=5.1,19.2 \mathrm{~Hz}, \mathrm{H}-6 \mathrm{a}), 2.45(1 \mathrm{H}, \mathrm{br}, J=13.2 \mathrm{~Hz}, \mathrm{H}-3 \mathrm{~b}), 2.20(1 \mathrm{H}, \mathrm{dt}, J=3.3,13.6$ $\mathrm{Hz}, \mathrm{H}-2 \mathrm{~b}), 2.00(1 \mathrm{H}, \mathrm{m}, \mathrm{H}-9), 1.75(1 \mathrm{H}, 1 \mathrm{H}$, br d, $J=12.5 \mathrm{~Hz}, \mathrm{H}-1 \mathrm{~b}), 1.71(1 \mathrm{H}, \mathrm{br} \mathrm{d}, J=13.5 \mathrm{~Hz}, \mathrm{H}-5), 1.56-1.61(1 \mathrm{H}, \mathrm{m}$, H-12b), 1.51-1.61 (2H, m, H-11b, H2a), 1.31 (3H, s, H-18), 1.25-1.31 (1H, m, H-12a), 1.06-1.13 (2H, m, H-1a, H-3a), 1.07 (3H, s, H-17), 0.95 (3H, s, H-20). ${ }^{13} \mathrm{C}-\mathrm{NMR}\left(125 \mathrm{MHz}\right.$, pyridine- $\left.d_{5}\right): \delta 199.8$ (C-7), 179.4 (C-19), 145.1 (C-15), $141.0(\mathrm{C}-14)$, 137.2 (C-8), 114.3 (C-16), 51.0 (C-5), 50.5 (C-9), 43.8 (C-10), 39.4 (C-6), 39.2 (C-13), 39.1 (C-1), 38.5 (C-3), 37.3 (C-4), 35.1 (C-12), 28.7 (C-18), 28.0 (C-2), 19.2 (C-11), 13.1 (C-20).

This should be replaced by:

7-oxo-sandaracopimaric acid (OSA)

Colorless needles: mp 247-249 ${ }^{\circ} \mathrm{C}$, EI-MS m/z: $316.4[\mathrm{M}+],{ }^{1} \mathrm{H}-\mathrm{NMR}\left(500 \mathrm{MHz}\right.$, pyridine- $\left.d_{5}\right): \delta 6.81(1 \mathrm{H}, \mathrm{s}, \mathrm{H}-14), 5.67(1 \mathrm{H}$, dd, $J=10.2,16.2 \mathrm{~Hz}, \mathrm{H}-15), 5.02(1 \mathrm{H}, \mathrm{dd}, J=1.4,10.2 \mathrm{~Hz}, \mathrm{H}-16 \mathrm{~b}), 4.80(1 \mathrm{H}, \mathrm{dd}, J=1.4,16.2 \mathrm{~Hz}, \mathrm{H}-16 \mathrm{a}), 3.72(1 \mathrm{H}, \mathrm{dd}, J=$ 13.6, 19.2 Hz, H-6b), 3.13 (1H, dd, $J=5.1,19.2 \mathrm{~Hz}, \mathrm{H}-6 \mathrm{a}), 2.45$ (1H, br, $J=13.2 \mathrm{~Hz}, \mathrm{H}-3 \mathrm{~b}), 2.20$ (1H, dt, $J=3.3,13.6 \mathrm{~Hz}, \mathrm{H}-$ 2b), $2.00(1 \mathrm{H}, \mathrm{m}, \mathrm{H}-9), 1.75(1 \mathrm{H}, 1 \mathrm{H}, \mathrm{br} \mathrm{d}, J=12.5 \mathrm{~Hz}, \mathrm{H}-1 \mathrm{~b}), 1.71(1 \mathrm{H}, \mathrm{br} \mathrm{d}, J=13.5 \mathrm{~Hz}, \mathrm{H}-5), 1.56-1.61(1 \mathrm{H}, \mathrm{m}, \mathrm{H}-12 \mathrm{~b})$, 1.51-1.61 (2H, m, H-11b, H2a), 1.31 (3H, s, H-19), 1.25-1.31 (1H, m, H-12a), 1.06-1.13 (2H, m, H-1a, H-3a), 1.07 (3H, s, H17), 0.95 (3H, s, H-20). ${ }^{13} \mathrm{C}-N M R ~\left(125 \mathrm{MHz}\right.$, pyridine- $\left.d_{5}\right): \delta 199.8$ (C-7), 179.4 (C-18), 145.1 (C-15), 141.0 (C-14), 137.2 (C8), 114.3 (C-16), 51.0 (C-5), 50.5 (C-9), 43.8 (C-10), 39.4 (C-6), 39.2 (C-13), 39.1 (C-1), 38.5 (C-3), 37.3 (C-4), 35.1 (C-12), 28.7 (C-18), 28.0 (C-17), 20.9 (C-2), 19.2 (C-11), 13.1 (C-20). 
Below please find the correct version of Figure 1 on page 510 :

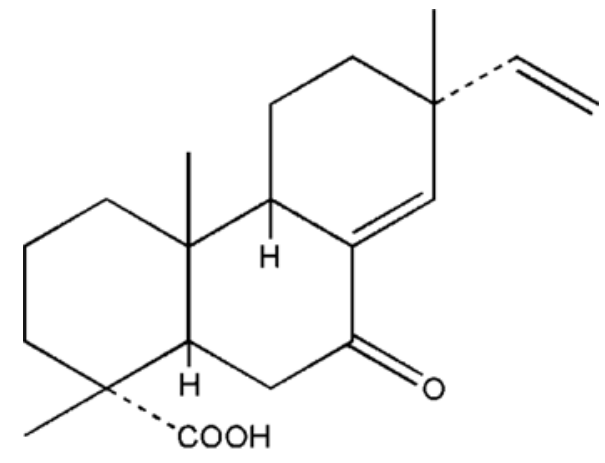

Error

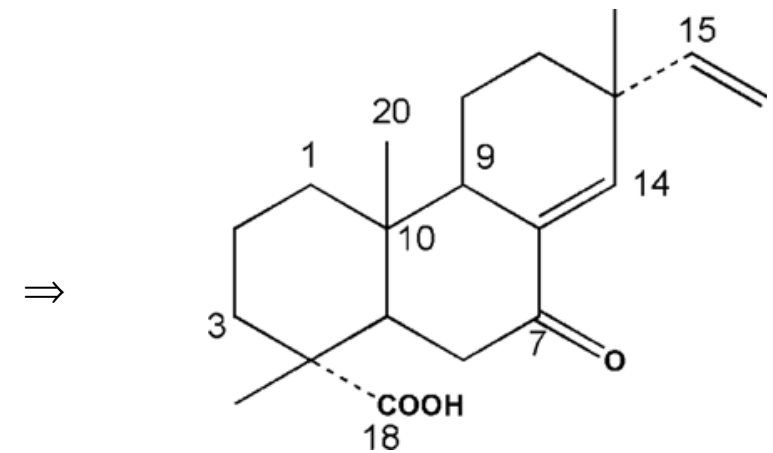

Correction

Fig. 1. Structure of 7-oxosandaracopimaric acid (OSA) 